\title{
LETTERATURA FEMMINILE IN MITTELEUROPA. UN BREVE CONFRONTO TRA SCRITTRICI EBREE, TRIESTINE E AUSTRIACHE.
}

\section{WOMEN'S LITERATURE IN CENTRAL EUROPE. A BRIEF COMPARISON BETWEEN JEWISH AUTHORS FROM TRIESTE AND AUSTRIA}

\begin{abstract}
Giovanna Neiger. Libera Università di Lingue e Comunicazione di Milano - Italia gneiger@hotmail.com
\end{abstract}

Riassunto: II contributo che scrittrici triestine e austriache di origine ebraica hanno rispettivamente offerto alla cultura otto-novecentesca è oggetto del presente lavoro.

Si sono prese in esame e sottoposte ad analisi comparativa le opere di autrici ebree triestine e di altrettante scrittrici ebree austriache. Tra le triestine, la cui identità ebraica si è affievolita, vi sono autrici che hanno svolto attività giornalistica, manifestato aspirazioni irredentistiche, scritto opere per un pubblico femminile e per l'infanzia, che non hanno superato l'usura del tempo. Diversamente dalle colleghe triestine, talune scrittrici austriache, femministe, giornaliste, pedagogiste o attiviste politiche hanno scritto testi che a tutt'oggi offrono spunti di riflessione.

Parole chiave: Scrittrici ebree triestine, scrittrici ebree austriache, giornaliste, femminismo, letteratura per l'infanzia.

\begin{abstract}
The object of this work is the contribution offered to the culture of XIX and XX centuries by women writers from Trieste and Austria. The works by Jewish authors from Trieste and Austria have been examined and a comparative analysis has been carried out. Among the women writers from Trieste, whose identity was wakened, there are authors who carried out journalism, shown irredentism aspiration, wrote works for women and for children that didn't overcome the passing of time. Unlike other colleagues from Trieste, some Austrian authors, who were feminists, journalists, pedagogists or political activists, wrote some text that, even nowadays, are offering some reflections.
\end{abstract}

Keywords: Women writers, Trieste, Austrian Jewish women writers, female journalists, feminism, children's literature. 


\section{Introduzione}

Amos Oz e Fania Oz-Salzberger, autori del volume Gli ebrei e le parole. Alle radici dell'identità ebraica, dedicano un ampio capitolo del loro saggio all'apporto che studiose e scrittrici ebree hanno offerto alla cultura.

Nei secoli passati le donne ebree, "sono state spesso zittite, proprio come quasi tutte le donne in tutte le altre culture" (Oz e Oz-Salzberger, 2013, p. 107) e di conseguenza si conservano le tracce di poche rappresentanti del genere femminile. Tra costoro è il caso di ricordare alcune personalità menzionate dal Talmud e precisamente Imma Shalom, Yalta e Beruria.

Di Imma Shalom, il cui nome significa "Madre di Pace", parla il Talmud Babilonese e ricorda di questa donna, sorella del rabbino Gamaliel II e moglie del rabbino Eliezer ben Hurcanus, una frase che riporta un insegnamento da lei ricevuto nella casa paterna. Dice Imma Shalom che tutte le porte che permettono di accedere all'intercessione divina sono sprangate ad eccezione di quelle a cui bussano coloro che hanno patito un torto. Sempre nel Talmud Babilonese si parla della dotta Yalta, moglie del rabbino Nachman, e di come scopri "che quando la Torah ci proibisce di fare o consumare qualcosa, ci permette sempre di fare o consumare qualcosa di abbastanza simile" (Oz e Oz-Salzberger, 2013, p. 102). Un'altra eccezionale figura femminile in grado di cimentarsi in dibattiti a cui prendono parte insigni studiosi è Beruria. Di costei si ricorda che insegnava "al marito a pregare per il ravvedimento dei malvagi, non per la loro fine" (Oz e Oz-Salzberger, 2013, p. 102).

Le tre donne sopra citate sono le capostipiti di una serie di ebree distintesi nel mondo della cultura e menzionate in Gli ebrei e le parole. Poche intellettuali mitteleuropee figurano nel testo e le più importanti sono Selma Stern, storica, Hannah Arendt, filosofa e poi tre scienziate, Lydia Rabinowitsch-Kempner, batteriologa, Lisa Meitner, insigne fisica e il medico Rahel Goitein Straus. Nel novero delle scrittrici spicca il nome di Hinde Esther Singer Kreitman sorella degli scrittori Israel Joshua e Isaac Bashevis Singer e la triestina Rachel Morpurgo. A quest'ultima poetessa, autrice di versi religiosi e laici in lingua ebraica, sono dedicate voci del Jüdisches Lexikon e della Jewish Encyclopedia online.

Ma nella Trieste che si affaccia sul mare e che, secondo Mauro Covacich (2011), i viennesi consideravano la città più meridionale dell'Europa del nord, Rachel Morpurgo non è l'unica intellettuale ebrea ad animare i salotti letterari e la vita culturale cittadina.

\section{Quadro teorico}

Alcune autrici triestine riscossero un discreto successo di pubblico, altre non ottennero da lettori e critica particolare attenzione. Tutte, con il trascorrere del tempo, sono state sommerse dall'oblio e pertanto il mio impegno è volto ora a riportarle alla 
memoria. Il loro talento letterario e artistico non è certo paragonabile a quello di autori coevi quali Svevo, Saba e Michelstaedter, ma la personalità di queste intellettuali ebree triestine presenta aspetti degni di essere restituiti alla nostra memoria.

Oltre a puntare l'attenzione sulle suddette letterate, intendo prendere in considerazione anche alcune scrittrici mitteleuropee, ebree germanofone. Mi propongo di confrontare i due gruppi di intellettuali al fine di individuare eventuali convergenze e/o divergenze di ordine biografico e letterario.

La prima scrittrice che presento è Ida Finzi che firmava i suoi scritti con lo pseudonimo Haydée, nome femminile di un personaggio del Don Giovanni di Byron nonché del Conte di Montecristo di Dumas. ${ }^{1}$

Haydée, figlia di un agiato borghese che amministra male i propri beni e sperpera il patrimonio familiare, è una giovane estroversa, brillante che vivacizza con la sua presenza i salotti triestini. Inizia a scrivere in giovanissima età, è infatti appena sedicenne quando nel 1883 un suo racconto viene pubblicato nel «Fanfulla della Domenica» di cui è direttore Ferdinando Martini. In quello stesso anno il suo primo breve pezzo giornalistico è pubblicato dal giornale irredentista «L'Indipendente». Per la giovane si profila una lunga e produttiva attività di pubblicista e infatti di lì a poco collabora all' «Illustrazione italiana» e anche «ll Piccolo» accoglierà i suoi articoli. Le suddette testate non sono le uniche ad avvalersi della collaborazione di Haydée, la cui firma appare su numerosi periodici: la «Nuova antologia», «La lettura» e altri ancora.

Oltre a dedicarsi all'attività giornalistica, Haydée scrive racconti, poesie, romanzi, opere teatrali, libri per l'infanzia. Partecipa a svariati concorsi letterari e fa incetta di premi. Anche il suo Libro della madre e del bambino, una guida per le mamme, ottiene un premio. II manuale è un'accozzaglia di stereotipi sessisti. Haydée inneggia alla sposa subordinata al marito, alla madre oblativa, alla casalinga che si sente realizzata solo quando svolge in casa le faccende domestiche e non nutre altre velleità. Inoltre, come osservano Roberto Curci e Gabriella Ziani (1993), I'autrice condanna "le maternità dolorose e colpevoli [...] le colpe materne" [e parla del] "lungo castigo" che inesorabile attende chi abbia avuto un figlio naturale. Ma nonostante i riconoscimenti ottenuti, la produzione letteraria di Haydée è finita nel dimenticatoio. Prima di passare a un'altra letterata triestina vorrei precisare che le norme di vita esposte nel Libro della madre e del bambino, Ida Finzi non le mise mai in pratica perché non si sposò né ebbe figli. Non si dedicò esclusivamente alle faccende domestiche, ma con il lavoro di giornalista e scrittrice aiutò la sua famiglia di origine che attraversava un periodo di ristrettezze economiche. Oltre a ciò Finzi svolse una mansione che

\footnotetext{
${ }^{1}$ Un altro nom de plume di Ida Finzi è «La signora in grigio» con cui era solita firmare i suoi articoli pubblicati sull'«lllustrazione italiana». Inoltre firma il suo romanzo La signorina di Montecristo, pubblicato a puntate nel giornale da lei diretto «La sera della domenica», con il nome d'arte F. Dehayé (F sta per Finzi e Dehayé è l'anagramma di Haydée).
} 
solitamente non è affidata a donne: diresse, anche se per pochi mesi, un settimanale illustrato «La sera della domenica».

Haydée conosceva e frequentava la conterranea Fortuna Morpurgo, in arte Willy Dias. Figlia di un benestante ebreo e di una cattolica, Fortuna è una giovane lettrice "forte" e un'appassionata scrittrice. I suoi interessi la spingono a intraprendere in giovane età la carriera giornalistica. Sono molte le testate a cui collabora: «Trieste letteraria», «ll Mattino», «ll Caffaro» e altre ancora. La sua firma è anche apparsa su «La Chiosa. Commenti settimanali femminili di vita politica e sociale» un periodico di cui è direttrice Flavia Steno, una valida professionista legata da amicizia a Willy Dias nonostante non fosse immune dal morbo antisemita. ${ }^{2}$ Molte altre donne lavorano, nella seconda metà dell'Ottocento, nelle redazioni di giornali e periodici tanto che il drammaturgo e critico Camillo Antona Traversi osserva come "le donnine nevrotiche, non senz'ingegno, di leggiera e svariata coltura, divise da mariti, separate dagli amanti, signorine e signore al tempo stesso, divennero a un tratto collaboratrici assidue de' migliori giornali letterari" (Antona Traversi, 1885, p. XX). Ma Fortuna Morpurgo non rispecchia l'immagine della "donnina" sola, poco equilibrata e di "leggiera e svariata cultura", che l'acida penna di Antona Traversi ha tratteggiato. Willy Dias è un'operosa lavoratrice: collabora a vari quotidiani e periodici affrontando tematiche legate alle vicende politiche del suo tempo, all'irredentismo, alla questione femminile. Contemporaneamente scrive romanzi rosa e con la sua attività letteraria si guadagna da vivere. È una scrittrice prolifica, i suoi libri riscuotono largo successo e vengono tradotti in varie lingue. Il pesco selvatico per esempio ebbe venticinque ristampe e duecentoquarantamila furono all'incirca le copie vendute. Agli inizi del Novecento la popolarità di Willy Dias era quasi pari a quella di Carolina Invernizio, Guido Da Verona e Liala.

Francesco De Nicola individua nei libri di Dias anche tematiche che si discostano dai consueti clichés della narrativa di consumo: "[Il sentiero fra le pietre e Sissignora] raccontano senza remore e con forti accenti critici le ingiustizie e i malesseri di una società per nulla rosa" (De Nicola, 2002, p. 30). Anche Giorgio Voghera nel saggio Presenza e spirito ebraici nella letteratura triestina ricorda "che furono ebree tre scrittrici che ebbero una certa notorietà: Ida Finzi (Haydée), Fortuna Morpurgo (Willy Dias) e Pia Rimini" (Voghera, 1980, p. 134). Ma nelle prime due letterate triestine citate da Voghera, lo spirito ebraico si è notevolmente affievolito e nell'ultima scrittrice è addirittura scomparso, in quanto Pia Rimini, figlia di un ebreo e di un'ebrea

\footnotetext{
${ }^{2} \mathrm{Nel}$ gennaio del 1920 i postelegrafonici indissero uno sciopero. Flavia Steno lo ritenne lesivo dei diritti degli utenti e attribui "le loro velleità, come sobillatori della società, alla diffusa appartenenza alla religione ebraica degli ideologi rivoluzionari che li guidano. Secondo [lei] da Marx a Lenin, da Lassalle a Liebknecht, da Engels a Rosa Luxemburg, da Bernstein a Trotski, da Treves a Ottolenghi, 'tutti guardano al sol dell'avvenire ma tengono le mani nelle casseforti dell'alta finanza internazionale israelita'". (Stolfi, 2007, p. 117)
} 
convertita al cattolicesimo, fu battezzata subito dopo la nascita e cercò di divenire una cattolica praticante.

Tornando a Willy Dias, Cristina Benussi vede confluire nella persona della scrittrice la straordinaria sintesi di due anime, quella cattolica e quella ebraica:

Sehr solide bleiben [in Sentiero fra le pietre] die Bindungen des Gehorsams zwischen Mutter und Sohn, der am Ende die Richtigkeit einer Entscheidung anerkennt, die darin bestand, eine schöne Frau vor allem aufgrund ihrer inneren Vorzüge geheiratet zu haben. Die beiden Seelen der Dias fließen dabei in eine ungewöhnliche Synthese der katholischen Vorstellung von Gehorsam und Opferbereitschaft mit der jüdischen von Opferbereitschaft und Projektorientiertheit ein, in die Figur einer Mutter, die arbeitet und sich ihre Rechte von der Kommunistischen Partei schützen läßt, der Dias 1945 auch beitritt. (Benussi, 2011, p. 275)

Quanto a Pia Rimini, la sua anima ebraica si è del tutto disciolta. La scrittrice confida solo nella religione cattolica. Nel suo romanzo /l diluvio fa un accenno agli ebrei e li descrive come una razza tarata, degenerata. Nonostante la sua sincera professione di fede cattolica e una adesione - intorno agli anni Trenta - al modello culturale di impronta fascista di donna subordinata all'vomo, Pia Rimini viene arrestata nel 1944 e successivamente internata ad Auschwitz da cui non uscirà viva. Dice Zygmunt Bauman che donne e uomini sono angustiati dal fatto di non saper quale identità scegliere e di dover essere sempre pronti a disfarsi della propria identità "nel caso [...] venga ritirata dal mercato o spogliata dei suoi poteri di seduzione" (Bauman, 2002, p. 186). Ma non a tutte le persone è concesso di scegliere, perché come osserva Voghera - quando al potere è arrivato Hitler egli "ha ribadito nelle menti di tutti noi il concetto che uno è ebreo anche quando non vuole esserlo" (Voghera, 1980, p. 137).

Altre autrici italiane di ascendenza ebraica meritano di essere ricordate. Rina del Prado, nom de plume di Vittoria Pardo, traduttrice e romanziera, collaborò a vari giornali, tra cui «Vittoria Colonna» un periodico di area cattolica rivolto a un pubblico femminile e scritto unicamente da donne. Carolina Coen Luzzatto, zia acquisita di Carlo Michelstaedter, fu autrice di poesie e opere teatrali per l'infanzia,. Non acquistò notorietà per le commedie morali ispirate a uno stile educativo non repressivo o per il suo salotto frequentato da personaggi illustri ma per la sua intensa attività di giornalista. Collaborò a testate locali firmando talvolta gli articoli con lo pseudonimo Arcolani, anagramma del suo nome, e fu direttore responsabile del «Corriere di Gorizia» dal 1883 al 1889, anno in cui il giornale venne chiuso. Due anni dopo assunse la direzione del "Corriere Friulano», incarico che conservò fino al 1914. Fu un'accesa irredentista come pure Enrica Barzilai Gentilli, ricordata ancor'oggi per Maternità, in cui affronta il tema del divorzio, a sua detta ammissibile unicamente se la separazione dei genitori possa essere vantaggiosa per i figli. Tullia Catalan in un suo saggio prende 
in esame la loro comune aspirazione irredentista all'unificazione con il regno d'Italia, giungendo a interessanti conclusioni:

Despite their different life experiences and their belonging to two different generations [...] these two border-region writers [...] breathed the climate of tension fed by the nationalist conflict between Slovenes and Italians [...] and this influenced and radicalized their Italian patriotism. In both of them, the idea became progressively entrenched that the internal enemy should be combated with all possible means, and the experience of war constituted the crucial turning point that marked, especially for Enrica, the embracing of strongly racist positions against the German enemy. [...] Thus, for both of them, the passage from anti-Slav prejudice, which is clearer in Carolina's case and also structured in her narratives and language, to a stereotyped image of the enemy that contained racist contours was a process that began at the turn from the nineteenth century to the twentieth and came to fruition during the war. (Catalan, 2020, p. 370)

Rivolgo un ultimo accenno a Elody Oblath, moglie di Giani Stuparich e amica di Scipio Slataper di cui tradusse in tedesco /l mio Carso, e autrice di lettere e pagine autobiografiche che ancor oggi attraggono i lettori.

Eugenie Schwarzwald è la prima autrice austriaca che intendo presentare. Schwarzwald fondò a Vienna una scuola per ragazze, un Mädchengymnasium, e si avvalse della collaborazione di prestigiosi docenti, quali Otto Rommel, Adolf Loos, Oskar Kokoschka e Arnold Schönberg. Era a conoscenza del pensiero dei maggiori pedagogisti del suo tempo quali Franz Cizek, Hermann Lietz e Maria Montessori e si ispirava ai loro dettami. Un clima gaio e rilassato, in cui lo scolaro si senta libero e possa studiare con piacere senza essere oppresso dalle insistenti richieste di insegnanti troppo esigenti, questo l'obiettivo che Schwarzwald intendeva perseguire. Nell'ambiente sereno del ginnasio femminile si formarono molte ragazze che divennero famose come Anna Freud, figlia di Sigmund; Alice Herdan-Zuckmayer, scrittrice e moglie di Carl Zuckmayer; Helene Weigel, attrice; Ruth Karplus, pittrice e stilista; Elsie Altmann-Loos, ballerina, e ancora tante altre professioniste.

L'instancabile Schwarzwald, oltre ad occuparsi della scuola, fu l'animatrice di un salotto letterario sito al numero 68 della Josefstädterstrasse e frequentato da illustri personaggi quali Rainer Maria Rilke, Karl Popper, Paul Lazarsfeld e Lou AndreasSalomé. Collaborò a parecchie testate giornalistiche, tra cui «Neue Freie Presse», «Wiener Allgemeine Zeitung», «Wiener Tag», «Neues Wiener Journal», «Neues Wiener Tagblatt», «Neue Zürcher Zeitung», «Zürcher Illustrierte», «Vossische Zeitung», «Aus fremden Zungen», «Frauenblatt» e altre ancora che pubblicarono sue opere di narrativa e saggistica. Schwarzwald fu un personaggio singolare: Musil, Kraus e Canetti, che la conobbero e frequentarono, la presero a modello per i personaggi delle loro opere. Musil, autore di Der Mann ohne Eigenschaften, si ispirò a lei per creare il personaggio di Diotima; Kraus, in Die letzten Tage der Menschheit descrive la signora 
Schwarz-Gelber, moglie di un consigliere di corte che ha le sembianze di Schwarzwald, e infine Canetti in Das Augenspiel. Lebensgeschichte 1931-1937, parla di una pedagogista che altri non è se non la fondatrice del viennese Mädchengymnasium.

L'operato di Schwarzwald riporta alla memoria l'attività di Bertha Pappenheim. A molti studiosi è nota Anna O., la giovane paziente del medico viennese Josef Brever, affetta da isteria di cui si parla in Studien über Hysterie, opera dello stesso Brever e di Freud, che risponde al nome di Bertha Pappenheim. Superato il disturbo psichico, la giovane Pappenheim, oltre a pubblicare poesie, racconti, un'opera teatrale, articoli giornalistici e tradurre in tedesco A Vindication of the Rights of Woman di Mary Wollstonecraft, si impegna in attività filantropiche e iniziative in favore delle donne. Nel 1904 fondò con le due insegnanti Sidonie Werner e Henriette May il Jüdischer Fravenbund che si impegnava tra l'altro di promuovere attività assistenziali, favorire l'attività professionale delle donne, combattere la prostituzione. A Pappenheim, che per venti anni diresse il Jüdischer Fravenbund, e alle sue collaboratrici va attribuito il merito di avere in parte realizzato importanti iniziative in favore delle sue correligionarie. A Neu-Isenburg, per esempio, riusci a creare un centro per figli illegittimi e per le loro madri. Sempre in tema di difesa dei diritti delle donne, va ricordata Adele Schreiber-Krieger, autrice di una biografia di un'illustre femminista: Hedwig Dohm als Vorkämpferin und Vordenkerin never Fravenideale. Schreiber-Krieger fu deputata della Sozialdemokratische Partei Deutschlands nel Parlamento tedesco dal 1920 al 1924 e dal 1928 al 1932 e in quegli anni lottò per la rivendicazione dei diritti economici, civili e politici delle donne. Con altre femministe si adoperò per la revisione dell'articolo 218 del Codice penale tedesco relativo all'interruzione di gravidanza.

Un'altra scrittrice degna di nota, la cui produzione letteraria spazia dal trattato sul cinema al libro di fisica nucleare spiegata ai bambini e la cui opera più famosa appartiene al genere letterario di narrativa per bambini, è la viennese Anna Maria Jokl che visse a Berlino, Praga e in Gran Bretagna, per poi stabilirsi definitivamente in Israele negli anni Sessanta. A Praga, Jokl fa la conoscenza di Oskar Kokoschka, che decide di scrivere la postfazione al primo libro dell'autrice, Die wirklichen Wunder des Basilius Knox. Ein Roman über Physik für Kinder von 10 bis 70 Jahren. La sua seconda opera è Die Perlmutterfarbe. Ein Kinderroman für fast alle Leute il cui manoscritto, completato a Praga dopo il 1937, è stato tratto in salvo in circostanze quasi miracolose durante la fuga dell'autrice attraverso il confine con la Polonia. Die Perlmutterfarbe viene finalmente pubblicato nella DDR nel 1947, e riscuote subito un gran successo. II romanzo, che narra la storia di una scuola che rispecchia come in un microcosmo l'impostazione dittatoriale nazifascista, è stato dapprima tradotto in lingua ceca e dopo cinque anni dalla sua pubblicazione in italiano, e in questa lingua più volte ritradotto, fino all'ultima edizione, curata da Paola Buscaglione Candela per la collana La Giuntina, nel 2006. Nelle scuole italiane, nel contesto educativo di una cultura della memoria e in particolare dell'annuale 
celebrazione della Giornata della Memoria, questo romanzo risulta essere tra quelli più letti, insieme al Diario di Anna Frank e ai libri di Lia Levi.

Jokl scriveva libri per l'infanzia perché in quei tempi confusi rappresentavano per lei una speranza non contaminata. In questa direzione va anche il messaggio che dà Kokoschka al termine della sua postfazione al Basilius Knox: date in mano ai vostri bambini dei libri dai quali possano imparare a giovare all'umanità: "Darum gebt Euren Kindern Bücher in die Hände, aus denen man lernt, wie man der ganzen Menschheit dienen kann" (Jokl, 1997, p. 266).

Ai problemi educativi si interessò anche Mira Lobe. Nata a Görlitz da genitori ebrei, Lobe contava ventitré anni quando si rifugiò nel 1936 in Palestina per sfuggire all'oppressione nazionalsocialista. Nel 1950 si stabili definitivamente a Vienna e si dedicò con passione alla sua attività preferita, la creazione letteraria perché per lei "das Schreiben ist nach der Liebe die zweitschönste Sache der Welt" (Ziegler, 2014), scrivere è, dopo l'amore, la cosa più bella del mondo. Lobe dunque non istituisce scuole, ma si occupa di letteratura per l'infanzia e nell'arco della sua vita pubblica circa un centinaio di volumi che sono stati tradotti in ben ventotto lingue. Nei suoi libri, dei veri long seller, con i quali ha ottenuto degli importanti premi e che a tutt'oggi troviamo esposti nelle librerie, l'autrice infonde nell'animo dei suoi piccoli lettori sani principi morali e sopra tutto il rispetto e l'amore per il prossimo.

Rose Ausländer, una scrittrice animata da un irrefrenabile impulso a poetare, non si rivolge ai ragazzi, ma agli adulti. E come tante sue correligionarie si sposta come una nomade. Ausländer dalla nativa Czernowitz approda a Vienna, poi a Budapest, e nuovamente a Czernowitz. Emigra in America poi torna in Europa a Bucarest e ancora nel ghetto di Czernowitz dove incontra Paul Celan.

Diversamente da Adorno, Ausländer è convinta che sia ancora possibile fare poesia dopo la Shoah:

\author{
Hoffnung IV \\ Mein \\ aus der Verzweiflung \\ geborenes Wort \\ aus der verzweifelten Hoffnung \\ daß Dichten \\ noch möglich sei
}

(Ausländer, 2013, pp. 90-91)

Aggiungo a questo breve elenco il nome di Venetiana Taubner-Calderon, meglio conosciuta come Veza Canetti. Negli anni Trenta Veza Canetti, moglie del premio 
Nobel Elias Canetti, pubblicò alcuni racconti su vari giornali firmandoli con diversi pseudonimi, Veza Magd, Veronika Knecht e Martha Murner ${ }^{3}$ Con I'avvento del nazismo si trasferì a Londra e continuò a scrivere, ma le sue opere rimasero inedite. Veza Canetti morì nel 1963, ma solo nel 1990, grazie allo studioso Helmuth Göbel e a Eckart Früh fu pubblicato un suo romanzo, cui han fatto seguito altre pubblicazioni, Die gelbe Straße in cui "tutte le figure femminili, siano esse mogli e madri, cameriere o ragazze in cerca di lavoro, vecchie e paralitiche, sono sempre costrette a soccombere in una realtà in cui solo il più forte sopravvive" (Bertocchi, 2006, p. 8).

\section{Conclusioni}

Sia le scrittrici triestine che quelle austriache provengono da ambienti borghesi e quasi tutte hanno svolto una intensa attività giornalistica, adottato uno pseudonimo e contano una ricca produzione letteraria rivolta all'infanzia.

Le peregrinazioni per vari paesi è una saliente caratteristica delle ebree mitteleuropee di lingua tedesca che le distingue dalle colleghe mitteleuropee italiane. Inoltre, a causa dell'ostinato antisemitismo dell'ambiente in cui vivono, le austriache hanno sviluppato loro malgrado un profondo senso identitario e sono molto impegnate nel versante socio umanitario. E non mancano le persone che prendono parte attiva alla vita politica. Per il loro impegno civile e politico non ottengono solo premi letterari ma anche riconoscimenti d'altro tipo come Ausländer, cui fu conferita la Croce al merito della Repubblica federale tedesca.

Le triestine sono irredentiste con forti sentimenti di italianità e una spiccata disposizione ad assimilarsi alla popolazione cattolica, invece le scrittrici di lingua tedesca di ascendenza ashkenazita e sefardita sanno mantenere un forte legame con le proprie radici.

Molte delle suddette autrici italiane e austriache, testimoni di un mondo dominato dalla furia nazista, nutrono l'indomita speranza di educare le nuove generazioni alla pacifica convivenza dei popoli, convinte che "la letteratura non può cambiare la realtà, ma può cambiare [l'] atteggiamento verso la realtà, verso l'arbitrarietà costituita dall'ordine violento e razzista"' (Grossman, 2000, p. 31).

Come afferma Rose Alison, va riconosciuto alle donne ebree austriache il merito di aver offerto un rilevante contributo alla cultura del loro paese: "[...] Jewish women played a key role in fin de siècle Vienna, both in terms of their participation in the various spheres of activity, which they pursued with enthusiasm and which served them in their sometimes challenging position as Jewish women in a less than

\footnotetext{
3 Bettina Bannasch ritiene che "hinter [ihnen] ein Konzept dienenden Magdtums steht" e non riesce a scorgere nell'opera di Veza Canetti "ein emanzipiertes oder gar feministisches Frauenbild" (Bannasch, 2002, p. 39).
} 
welcoming climate, and in terms of the imagined Jewish woman found in a wide array of works by Jewish (and non-Jewish) men" (Alison, 2008, p. 219).

\section{Bibliografia}

Alison, R. (2008). Jewish Women in Fin de Siècle Vienna. Austin: University of Texas Press.

Ausländer, R. (2013). Hoffnung IV. In B. Nolz, W. Popp, Leben im Zeichen von Verfolgung und Hoffnung. Jüdische Autorinnen und Autoren in der neveren deutschen Literatur (pp. 90-91). Berlin, Alemania: LIT Verlag.

Bannasch, B. (2002). Zittern als eine Bewegung des Widerstands: Veza Canettis frühe Erzählungen "Geduld bringt Rosen" und der Roman „Die Gelbe Straße". In H. L. Arnold, H. Göbel (a cura di): Veza Canetti (pp. 30-47). München, Alemania: edition text + kritik.

Bauman, Z. (2002). La società individualizzata (trad. di Giovanni Arganese). Bologna, Italia: il Mulino.

Benussi, C. (2011). Schrift, Weiblichkeit und kulturelle Identität. Jüdische Schriftstellerinnen Triests im 20. Jahrhundert. In A. A. Wallas (a cura di), Jüdische Identitäten in Mitteleuropa. Literarische Modelle der Identitätskonstruktion (pp. 269-282). Tübingen, Alemania: Niemeyer.

Bertocchi, M. (2006). La lingua salvata di Veza Canetti. Vita e opere di una scrittrice viennese. Milano, Italia: CUEM.

Catalan, T. (2020). The Construction of the Enemy in Two Jewish Writers: Carolina Coen Luzzatto and Enrica Barzilai Gentilli. In M. Baumeister, Ph. Lenhard e R. Nattermann. (a cura di), Rethinking the Age of Emancipation. Comparative and Transnational Perspectives on Gender, Family and Religion in Italy and Germany, 1800-1918. New York, EEUU, Oxford, Inglaterra: Berghahn Books.

Covacich, M. (19 agosto 2011). Trieste, la Mitteleuropa anomala. l'Espresso. Estratto da https://espresso.repubblica.it/visioni/cultura/2011/08/19/news/trieste-lamitteleuropa-anomala-1.34474

Curci, R., Ziani, G. (1993). Bianco, rosa e verde. Scrittrici a Trieste fra '80o e '90o. Trieste, Italia: Lint.

De Nicola, F., Zannoni P. A. (2002). La fama e il silenzio. Scrittrici dimenticate del primo Novecento. Venezia, Italia: Marsilio.

Grossman, D. (2000). La memoria della Shoah. Intervista di Matteo Bellinelli. Bellinzona: Casagrande.

Herlitz, G. Kirschner, B. (1930). Jüdisches Lexikon. Ein enzyklopädisches Handbuch des jüdischen Wissens in vier Bänden. Berlin, Alemania: Jüdischer Verlag.

Jokl, A.M. (1997). Die wirklichen Wunder des Basilius Knox. Ein Roman über die Physik für Kinder von 10 bis 70 Jahren. Nachwort von Oskar Kokoschka. Frankfurt/Main, Leipzig, Alemania: Insel Verlag.

Oz, A., Oz-Salzberger, F. (2013). Gli ebrei e le parole. Alle radici del'identità ebraica (trad. di Elena Loewenthal). Milano, Italia: Feltrinelli.

Stolfi, V. (2007). La collaborazione giornalistica di Flavia Steno con il "Secolo XIX" e "La Chiosa", Milano, Italia: Lampi di Stampa. 
Antona Traversi, C. (1885). Prefazione. In V. Peri, Della critica letteraria moderna. Bologna, Italia: Zanichelli.

Voghera, G. (1980). Gli anni della psicanalisi. Pordenone, Italia: Studio Tesi.

Ziegler, M. (8 giugno 2014). Sie hätte noch Ideen gehabt. Wiener Zeitung. Estratto da https://www.wienerzeitung.at/nachrichten/reflexionen/vermessungen/574998Sie-haette-noch-Ideen-gehabt.html?em_cnt_page=1

\section{Cómo referenciar este artículo/How to reference this article:}

Neiger, G. (2021). Letteratura femminile in mitteleuropa. Un breve confronto tra scrittrici ebree, triestine e austriache. iQUAL. Revista de Género e Igualdad, 4, 161-171, doi: 10.6018/iqual. 442821.

Neiger, G. (2021). Letteratura femminile in mitteleuropa. Un breve confronto tra scrittrici ebree, triestine e austriache.. [Women's literature in central Europe. A brief comparison between jewish authors from Trieste and Austria]. iQUAL. Revista de Género e Igualdad, 4, 161-171, doi: 10.6018/iqual. 442821 